5-1-2014

\title{
Inference for the Rayleigh Distribution Based on Progressive Type-II Fuzzy Censored Data
}

\author{
Abbas Pak \\ Shahid Chamran University, Ahvaz, Iran, a-pak@scu.ac.ir \\ Gholam Ali Parham \\ Shahid Chamran University, Ahvaz, Iran, Parham_g@scu.ac.ir \\ Mansour Saraj \\ Shahid Chamran University, Ahvaz, Iran, seraj.a@scu.ac.ir
}

Follow this and additional works at: http://digitalcommons.wayne.edu/jmasm

Part of the Applied Statistics Commons, Social and Behavioral Sciences Commons, and the Statistical Theory Commons

\section{Recommended Citation}

Pak, Abbas; Parham, Gholam Ali; and Saraj, Mansour (2014) "Inference for the Rayleigh Distribution Based on Progressive Type-II Fuzzy Censored Data," Journal of Modern Applied Statistical Methods: Vol. 13 : Iss. 1 , Article 19.

DOI: $10.22237 /$ jmasm/1398917880

Available at: http://digitalcommons.wayne.edu/jmasm/vol13/iss1/19

This Regular Article is brought to you for free and open access by the Open Access Journals at DigitalCommons@WayneState. It has been accepted for inclusion in Journal of Modern Applied Statistical Methods by an authorized editor of DigitalCommons@WayneState. 


\section{Inference for the Rayleigh Distribution Based on Progressive Type-II Fuzzy Censored Data}

\author{
Abbas Pak \\ Shahid Chamran University \\ Ahvaz, Iran
}

\author{
Gholam Ali Parham \\ Shahid Chamran University \\ Ahvaz, Iran
}

\author{
Mansour Saraj \\ Shahid Chamran University \\ Ahvaz, Iran
}

Classical statistical analysis of the Rayleigh distribution deals with precise information. However, in real world situations, experimental performance results cannot always be recorded or measured precisely, but each observable event may only be identified with a fuzzy subset of the sample space. Therefore, the conventional procedures used for estimating the Rayleigh distribution parameter will need to be adapted to the new situation. This article discusses different estimation methods for the parameters of the Rayleigh distribution on the basis of a progressively type-II censoring scheme when the available observations are described by means of fuzzy information. They include the maximum likelihood estimation, highest posterior density estimation and method of moments. The estimation procedures are discussed in detail and compared via Monte Carlo simulations in terms of their average biases and mean squared errors. Finally, one real data set is analyzed for illustrative purposes.

Keywords: $\quad$ Progressive type-II censoring, fuzzy information, maximum likelihood principle, highest posterior density estimation

\section{Introduction}

The Rayleigh distribution was originally introduced by Lord Rayleigh (1880) in the field of acoustics; since its introduction, many researchers have used the distribution in different fields of science and technology. The Rayleigh distribution is frequently used to model wave heights in oceanography, in communication engineering and it also has a wide application in lifetime data analysis, especially in reliability theory and survival analysis. An important characteristic of the Rayleigh distribution is that its hazard rate is a linearly increasing function of time at constant rate, which makes it a suitable model for

Abbas Pak is in the Department of Statistics. Email at: a-pak@scu.ac.ir. Gholam Ali Parham is in the Department of Mathematics. Email at: parham_g@scu.ac.ir.Mansour Saraj is in the Department of Mathematics. Email at: seraj.a@scu.ac.ir. 


\section{INFERENCE FOR THE RAYLEIGH DISTRIBUTION}

the lifetime of components/items that age rapidly with time. Thus, as time increases, the reliability function of the Rayleigh distribution decreases at a much higher rate than the exponential reliability function does. The probability density function (pdf) and the cumulative distribution function (cdf) of a Rayleigh random variable $X$ can be written as:

$$
f(x)=2 \lambda x e^{-\lambda x^{2}} ; \quad x>0, \lambda>0,
$$

and

$$
F(x)=1-e^{-\lambda x^{2}} ; \quad x>0,
$$

respectively. Inferences for the Rayleigh distribution have been discussed by several authors. Dyer and Whisenand (1973) demonstrated the importance of this distribution in communication engineering. Bhattacharya and Tyagi (1990) mentioned that in some clinical studies dealing with cancer patients, the survival pattern follows the Rayleigh distribution. Chung (1995) obtained the best invariant estimator and the Bayes estimator of the parameter of Rayleigh distribution under entropy loss. Fernandez (2010) addressed the problems of estimating the parameter, hazard rate and reliability function of the Rayleigh distribution on the basis of sample quantiles. Dey and Maiti (2012) derived Bayes estimator of the Rayleigh parameter and its associated risk based on extended Jeffrey's prior.

In many life testing and reliability experiments, a sample of $n$ items is tested, and the experiment is terminated when all of them fail. This procedure may take a long time when the lifetime distribution of items has a thick tail. Moreover, if the items are expensive, such as medical equipment, it is costly to gather information from the whole sample. There are many situations where experimental units are lost or removed from the test before complete failure. For example, individuals in a clinical trial may drop out of the study, the study may have to be terminated early for lack of funds or the test units may accidentally break. In other scenarios, the experiment may have to be terminated in order to free up testing facilities for other purposes.

In view of above, censoring is used in life testing to save time and cost of testing units. The removal of units in a test may be unintentional or pre-planned. Data obtained from such experiments are called censored sample. There are many types of censoring schemes used in lifetime analysis. The two most common 


\section{PAK ET AL}

censoring schemes are termed type-I and type-II censoring schemes. In the conventional type-I censoring scheme, the experiment continues up to a prespecified time $T$; the conventional type-II censoring scheme requires the experiment to continue until a pre-specified number of failures occur. These schemes, however, do not allow removal of units before the termination of the experiment; thus, a more general kind of censoring scheme called progressive type-II censoring is considered, which is as follows: Suppose that $n$ units are placed on a life test and the experimenter decides beforehand a quantity $m$, the number of units to be failed. Now at the time of the first failure, $R_{1}$ of the remaining $n-1$ surviving units are randomly removed from the experiment. Continuing on, at the time of the second failure, $R_{2}$ of the remaining $n-R_{1}-2$ units are randomly removed from the experiment. Finally, at the time of the $m^{\text {th }}$ failure, all the remaining $n-m-R_{1}-\ldots-R_{\mathrm{m}-1}\left(=R_{\mathrm{m}}\right)$ surviving units are removed from the experiment. The work on progressive censoring has become popular in life-testing and reliability studies. Kim and Han (2009) studied the problem of estimating the scale parameter of the Rayleigh distribution under general progressive censoring. Krishna and Kumar (2011) discussed reliability estimation for the Lindley distribution with progressive type-II censored data. Lee et al. (2011) obtained a Bayes estimator under the Rayleigh distribution with a progressive type-II right censored sample. Raqab and Madi (2011) addressed inference for the generalized Rayleigh distribution based on progressively censored data. Azimi et al. (2012) considered the Bayesian estimation of the parameter and reliability function of Rayleigh distribution based on a progressively type-II censored sample. Rastogi and Tripathi (2012) studied parameter estimation of the Burr type XII distribution on the basis of a progressively type-II censored sample. Pradhan and Kundu (2009) considered the statistical inference of the unknown parameters of the generalized exponential distribution in presence of progressive censoring. A recent account on progressive censoring schemes can be obtained in the monograph by Balakrishnan and Aggarwala (2000) or in the excellent review article by Balakrishnan (2007).

The above referenced studies for estimating parameters of different lifetime distributions under progressive type-II censoring are limited to precise data. However, in real world situations, experiments do not provide exact information. For example, the reaction time of a person to a certain stimulus in a psychological experience cannot be exactly determined, but the psychologist is able to determine it by means of the following imprecise information, such as: The time of reaction is approximately 25 to 35 seconds. To deal with the lack of precision of the data, it is necessary to incorporate the fuzzy concept to statistical techniques. Recently, 


\section{INFERENCE FOR THE RAYLEIGH DISTRIBUTION}

Pak et al. (2013) proposed a new method to determine the maximum likelihood estimate of the scale parameter of a Rayleigh distribution under doubly type-II censored sample from fuzzy data. Further, in a life testing experiment, some test units may need to be removed at different stages in the study for various reasons. This would lead to progressive censoring. The purpose of this article is to develop the inferential procedures for the Rayleigh distribution under a progressive type-II censoring scheme when the available observations are reported by means of fuzzy information. The maximum likelihood estimate (MLE) of the parameter $\lambda$ is obtained by using EM algorithm and the highest posterior density (HPD) estimate of the unknown parameter is computed. The estimation via method of moments is discussed, a Monte Carlo simulation study is presented, and a comparison of all estimation procedures developed and one real data set is analyzed for illustrative purposes.

First, the fundamental notation and basic definitions of fuzzy set theory used herein is reviewed. Consider an experiment characterized by a probability space $S=\left(\Omega, F, P_{\theta}\right)$ where $(\Omega, F)$ is a measurable space and $P_{\theta}$ belongs to a specified family of probability measures $\left\{P_{\theta}, \theta \in \Theta\right\}$ on $(\Omega, F)$. Assume that the observer cannot distinguish or transmit with exactness the outcome in the performance of $S$, but that rather the available observation may be described in terms of fuzzy information, which is defined as:

\section{Definition 1}

A fuzzy event $\tilde{\omega}$ on $\Omega$, characterized by a Borel measurable membership function $\mu_{\tilde{\omega}}(\omega)$ from $\Omega$ to $[0,1]$, where $\mu_{\tilde{\omega}}(\omega)$ represents the grade of membership of $\omega$ to $\tilde{\omega}$, is called fuzzy information associated with the experiment $\mathcal{S}$.

The set consisting of all observable events from the experiment $\mathcal{S}$ determines a fuzzy information system associated with it, which is defined as:

\section{Definition 2}

A fuzzy information system (f.i.s.) $\tilde{\mathcal{S}}$ associated with the experiment $\mathcal{S}$ is a fuzzy partition $\left\{\tilde{\omega}_{1}, \ldots, \tilde{\omega}_{K}\right\}$ of $\mathcal{S}$, i.e., a set of $K$ fuzzy events on $\mathcal{S}$ satisfying the orthogonality condition (see Tanaka et al., 1979): 


\section{PAK ET AL}

$$
\sum_{k=1}^{K} \mu_{\tilde{\omega}_{k}}(\omega)=1
$$

where $\mu_{\tilde{\omega}_{k}}$ denotes the membership function of $\tilde{\omega}_{k}$.

According to Zadeh (1968), given the experiment $\mathcal{S}=\left(\Omega, F, P_{\theta}\right), \theta \in \Theta$, and a f.i.s. $\tilde{\mathcal{S}}$ associated with it, each probability measure $P_{\theta}$ on $(\Omega, F)$ induces a probability measure on $\tilde{\mathcal{S}}$ defined as:

\section{Definition 3}

The probability distribution on $\tilde{\mathcal{S}}$ induced by $P_{\theta}$ is the mapping $P$ from $\tilde{\mathcal{S}}$ to $[0,1]$ such that

$$
\mathrm{P}(\tilde{\omega})=\int_{\Omega} \mu_{\tilde{\omega}}(\omega) d P_{\theta}(\omega), \quad \tilde{\omega} \in \tilde{\mathcal{S}}
$$

In particular, the conditional density of a continuous random variable $\boldsymbol{Y}$ with p.d.f. $g(\boldsymbol{y})$ given the fuzzy event $\tilde{\boldsymbol{\omega}}$ can be defined as

$$
g(\boldsymbol{y} \mid \tilde{\omega})=\frac{\mu_{\tilde{\omega}}(\boldsymbol{y}) g(\boldsymbol{y})}{\int \mu_{\tilde{\omega}}(\boldsymbol{u}) g(\boldsymbol{u}) d \boldsymbol{u}}
$$

In order to model imprecise lifetimes, a generalization of real numbers is necessary. These lifetimes can be represented by fuzzy numbers. A fuzzy number is a subset, denoted by $\tilde{x}$, of the set of real numbers (denoted by $\mathbb{R}$ ) and is characterized by the so called membership function $\mu_{\tilde{x}}$ (.). Fuzzy numbers satisfy the constraints (Dubois and Prade, 1980):

1. $\mu_{\tilde{x}}: \mathbb{R} \rightarrow[0,1]$ is Borel-measurable;

2. $\exists x_{0} \in \mathbb{R}: \mu_{\tilde{x}}\left(x_{0}\right)=1$; and 


\section{INFERENCE FOR THE RAYLEIGH DISTRIBUTION}

3. The so-called $\lambda-$ cuts $(0<\lambda \leq 1)$, defined as $B_{\lambda}(\tilde{x})=\left\{x \in \mathbb{R}: \mu_{\tilde{x}}(x) \geq \lambda\right\}$, are all closed interval, i.e., $B_{\lambda}(\tilde{x})=\left[a_{\lambda}, b_{\lambda}\right], \forall \lambda \in(0,1]$.

Among the various types of fuzzy numbers, the triangular and trapezoidal fuzzy numbers are most convenient and useful in describing fuzzy lifetime data. The triangular fuzzy number can be defined as $\tilde{x}=(a, b, c)$ and its membership function is defined by the following expression:

$$
\mu_{\tilde{x}}(x)= \begin{cases}\frac{x-a}{b-a} & a \leq x \leq b, \\ \frac{c-x}{c-b} & b \leq x \leq c \\ 0 & \text { otherwise. }\end{cases}
$$

The trapezoidal fuzzy number can be defined as $\tilde{x}=(a, b, c, d)$ with the membership function:

$$
\mu_{\tilde{x}}(x)= \begin{cases}\frac{x-a}{b-a} & a \leq x \leq b, \\ 1 & b \leq x \leq c \\ \frac{d-x}{d-c} & c \leq x \leq d, \\ 0 & \text { otherwise }\end{cases}
$$

\section{Maximum likelihood estimation}

Suppose that $n$ identical units are put on a life testing experiment and that the lifetime distribution of each unit is given by (1). Prior to the experiment, a number $m<n$ is determined and the censoring scheme $\left(R_{1}, \ldots, R_{m}\right)$ with $R_{i} \geq 0$ and $\sum_{i=1}^{m} R_{i}+m=n$ is specified. Let $\boldsymbol{x}=\left(x_{1}, \ldots, x_{m}\right)$ denote the corresponding progressively type-II censored sample. The likelihood function for the parameter $\lambda$ becomes proportional to 


$$
L(\boldsymbol{x} ; \lambda)=\lambda^{m} e^{-\lambda \sum_{i=1}^{m}\left(1+R_{i}\right) x_{i}^{2}}
$$

Now consider the problem where $\boldsymbol{x}$ is not observed precisely and only partial information about $\boldsymbol{x}$ is available in the form of fuzzy numbers $\tilde{x}_{i}=\left(a_{i}, c_{i}, b_{i}\right), i=1, \ldots, m$, with the corresponding membership functions $\mu_{\tilde{x}_{1}}\left(x_{1}\right), \ldots, \mu_{\tilde{x}_{m}}\left(x_{m}\right)$. Let $c_{(1)} \leq c_{(2)} \leq \ldots \leq c_{(m)}$ denote the ordered values of the means of these fuzzy numbers. The lifetime of $R_{i}$ surviving units, which are removed from the test after the $i^{\text {th }}$ failure, can be encoded as fuzzy numbers $\tilde{z}_{i 1}, \ldots, \tilde{z}_{i R_{i}}$ with the membership functions

$$
\mu_{\bar{z}_{i j}}(z)=\left\{\begin{array}{ll}
0 & z \leq c_{(i)} \\
1 & z>c_{(i)}
\end{array}, \quad j=1, \ldots, R_{i} .\right.
$$

The fuzzy data $\tilde{\boldsymbol{w}}=\left(\tilde{x}_{1}, \ldots, \tilde{x}_{m}, \tilde{z}_{1}, \ldots, \tilde{z}_{m}\right)$ where $\tilde{z}_{i}$ is a $1 \times R_{i}$ vector with $\tilde{z}_{i}=\left(\tilde{z}_{i 1}, \ldots, \tilde{z}_{i R_{i}}\right)$ for $i=1, \ldots, m$, is thus the set of observed lifetimes. The corresponding observed data log-likelihood function can be obtained by using the expression (3) as follows:

$$
\begin{aligned}
L_{O}(\tilde{\boldsymbol{w}} ; \lambda) & =\sum_{i=1}^{m} \log \int 2 \lambda x e^{-\lambda x^{2}} \mu_{\tilde{x}_{i}}(x) d x+\sum_{i=1}^{m} \sum_{j=1}^{R_{i}} \log \int 2 \lambda z e^{-\lambda z^{2}} \mu_{\bar{z}_{\tilde{i}}}(z) d z \\
& =m \log \lambda+\sum_{i=1}^{m} \log \int 2 x e^{-\lambda x^{2}} \mu_{\tilde{x}_{i}}(x) d x-\lambda \sum_{i=1}^{m} R_{i} c_{(i)}^{2} .
\end{aligned}
$$

The maximum likelihood estimate of the parameter $\lambda$ can be obtained by maximizing the $\log$-likelihood $L_{O}(\tilde{\boldsymbol{w}} ; \lambda)$. Equating the partial derivative of the $\log$-likelihood (6) with respect to $\lambda$ to zero, the resulting equation is:

$$
\frac{\partial L_{O}(\tilde{\boldsymbol{w}} ; \lambda)}{\partial \lambda}=\frac{m}{\lambda}-\sum_{i=1}^{m} \frac{\int x^{3} e^{-\lambda x^{2}} \mu_{\tilde{x}_{i}}(x) d x}{\int x e^{-\lambda x^{2}} \mu_{\tilde{x}_{i}}(x) d x}-\sum_{i=1}^{m} R_{i} c_{(i)}^{2}=0 .
$$

Because there is no closed form of the solution to equation (7), an iterative numerical search can be used to obtain the MLE. The Expectation Maximization (EM) algorithm is a broadly applicable approach to the iterative computation of maximum likelihood estimates and useful in a variety of incomplete-data 


\section{INFERENCE FOR THE RAYLEIGH DISTRIBUTION}

problems. Because the observed fuzzy data $\tilde{\boldsymbol{w}}$ can be seen as an incomplete specification of a complete data, the EM algorithm is applicable to obtain the maximum likelihood estimate of the parameter. In the following, the fuzzy EM algorithm (see Denoeux, 2011) is used to determine the MLE of $\lambda$.

First, denote the lifetimes of the failed and censored units by $\boldsymbol{X}=\left(X_{1}, \ldots, X_{m}\right)$ and $\boldsymbol{Z}=\left(\boldsymbol{Z}_{1}, \ldots, \boldsymbol{Z}_{\boldsymbol{m}}\right)$, respectively, where $\boldsymbol{Z}_{\boldsymbol{i}}$ is a $1 \times R_{i}$ vector with $\boldsymbol{Z}_{\boldsymbol{i}}=\left(Z_{i 1}, \ldots, Z_{i R_{i}}\right)$, for $i=1, \ldots, m$. The combination of $\boldsymbol{W}=(\boldsymbol{X}, \boldsymbol{Z})$ forms the complete lifetimes and the corresponding log-likelihood function is denoted by $L_{c}(\boldsymbol{W}, \lambda)$, then, ignoring the additive constant,

$$
L_{c}(\boldsymbol{W}, \lambda)=n \log \lambda-\lambda\left\lfloor\sum_{i=1}^{m} x_{i}^{2}+\sum_{i=1}^{m} \sum_{j=1}^{R_{i}} z_{i j}^{2}\right\rfloor
$$

For the E-step, it is necessary to compute the pseudo log-likelihood function. It can be obtained from (8) as follows:

$$
n \log \lambda-\lambda\left\lfloor\sum_{i=1}^{m} E_{\lambda}\left(X_{i}^{2} \mid \tilde{x}_{i}\right)+\sum_{i=1}^{m} \sum_{j=1}^{R_{i}} E_{\lambda}\left(Z_{i j}^{2} \mid \tilde{z}_{i j}\right)\right\rfloor
$$

By using (4), the conditional expectations $E_{\lambda}\left(X_{i}^{2} \mid \tilde{x}_{i}\right)$ and $E_{\lambda}\left(Z_{i j}^{2} \mid \tilde{z}_{i j}\right)$ can be computed as:

$$
\begin{aligned}
& E_{\lambda}\left(X_{i}^{2} \mid \tilde{x}_{i}\right)=\frac{\int x^{3} e^{-\lambda x^{2}} \mu_{\tilde{x}_{i}}(x) d x}{\int x e^{-\lambda x^{2}} \mu_{\tilde{x}_{i}}(x) d x}, i=1, \ldots, m, \\
& E_{\lambda}\left(Z_{i j}^{2} \mid \tilde{z}_{i j}\right)=c_{(i)}^{2}+\frac{1}{\lambda}, i=1, \ldots, m, j=1, \ldots, R_{i} .
\end{aligned}
$$

Next, the M-step involves the maximization of the pseudo function (9). Therefore, if at the $h^{\text {th }}$ stage, the estimate of $\lambda$ is $\lambda^{(h)}$, then $\lambda^{(h+1)}$ can be obtained by maximizing

$$
L_{c}^{*}(\boldsymbol{W}, \lambda)=n \log \lambda-\lambda\left\lfloor\sum_{i=1}^{m} E_{\lambda^{(h)}}\left(X^{2} \mid \tilde{x}_{i}\right)+\sum_{i=1}^{m} \sum_{j=1}^{R_{i}} E_{\lambda^{(h)}}\left(Z^{2} \mid \tilde{z}_{i j}\right)\right\rfloor
$$


with respect to $\lambda$. From

$$
\begin{gathered}
\frac{\partial}{\partial \lambda} L_{c}^{*}(\boldsymbol{W}, \lambda)=0 \\
\hat{\lambda}^{(h+1)}=\frac{n}{\sum_{i=1}^{m}\left[E_{\lambda^{(h)}}\left(X^{2} \mid \tilde{x}_{i}\right)+R_{i}\left(c_{(i)}^{2}+1 / \lambda^{(h)}\right)\right]}
\end{gathered}
$$

The iteration process continues until convergence, i.e., until $\left|L_{O}\left(\tilde{\boldsymbol{w}} ; \lambda^{(h+1)}\right)-L_{O}\left(\tilde{\boldsymbol{w}} ; \lambda^{(h)}\right)\right|<\varepsilon$ for some pre-fixed $\varepsilon>0$.

\section{HPD estimation}

Consider the highest posterior density (HPD) estimation of the Rayleigh parameter based on observed fuzzy sample $\tilde{\boldsymbol{w}}$. As a conjugate prior for $\lambda$, take the $\operatorname{Gamma}(a, b)$ density with pdf given by

$$
\pi(\lambda)=\frac{b^{a}}{\Gamma(a)} \lambda^{a-1} e^{-\lambda b}, \quad \lambda>0
$$

where $a>0$ and $b>0$. Based on this prior, the posterior density function of $\lambda$ given the data can be written as follows:

$$
\pi(\lambda \mid \tilde{\boldsymbol{w}}) \propto \lambda^{m+a-1} e^{-\lambda\left(b+\sum_{i=1}^{m} R_{i} c_{(i)}^{2}\right)} \prod_{i=1}^{m} \int x e^{-\lambda x^{2}} \mu_{\tilde{x}_{i}}(x) d x
$$

The method of HPD estimation then estimates $\lambda$ as the mode of the posterior density $\pi(\lambda \mid \tilde{\boldsymbol{w}})$; therefore, the HPD estimate of $\lambda$ can be obtained by solving the equation

$$
\frac{\partial \log \pi(\lambda \tilde{\boldsymbol{w}})}{\partial \lambda}=0
$$

where 


\section{INFERENCE FOR THE RAYLEIGH DISTRIBUTION}

$$
\frac{\partial \log \pi(\lambda \mid \tilde{\boldsymbol{w}})}{\partial \lambda}=\frac{m+a-1}{\lambda}-b-\sum_{i=1}^{m} R_{i} c_{(i)}^{2}-\sum_{i=1}^{m} \frac{\int x^{3} e^{-\lambda x^{2}} \mu_{\tilde{x}_{i}}(x) d x}{\int x e^{-\lambda x^{2}} \mu_{\tilde{x}_{i}}(x) d x}
$$

However, the solution cannot be obtained explicitly. Theorem 1 discusses the existence and uniqueness of the HPD estimate of $\lambda$.

\section{Theorem 1}

Let $g(\lambda)$ denote the function on the right-hand side of the expression in (15). Then the root of the equation $g(\lambda)=0$ exists and is unique.

Proof. From (15), it is seen that $\lim _{\lambda \rightarrow 0} g(\lambda)=\infty$. Also, note that $g(\lambda)<\frac{m+a-1}{\lambda}, \forall \lambda \in(0, \infty)$, and consequently

$$
\lim _{\lambda \rightarrow 0} g(\lambda)<\lim _{\lambda \rightarrow 0} \frac{m+a-1}{\lambda}=0, \forall \lambda \in(0, \infty)
$$

Thus, the equation $g(\lambda)=0$ has at least one root in $(0, \infty)$. To prove that the root is unique, consider the first derivative of $g, g^{\prime}(\lambda)$ given by

$$
g^{\prime}(\lambda)=-\frac{m+a-1}{\lambda^{2}}+\sum_{i=1}^{m} \frac{\partial^{2}}{\partial \lambda^{2}} \log \int 2 x e^{-\lambda x^{2}} \mu_{\tilde{x}_{i}}(x) d x
$$

Because the integrand of the second term in (16) is a log-concave function of $\lambda$, and $g^{\prime}(\lambda)<0$. It follows that $g$ is a strictly decreasing function w.r.t. $\lambda$ and hence the equation $g(\lambda)=0$ has exactly one solution. The HPD estimate of $\lambda$ must be derived numerically. In the following, the Newton-Raphson algorithm to determine the HPD estimate is described.

The Newton-Raphson algorithm is a direct approach for estimating the relevant parameters in a likelihood function. In this algorithm, the solution of the likelihood equation is obtained through an iterative procedure. Let $\hat{\lambda}^{(h)}$ be the parameter value from the $h^{\text {th }}$ step. Then, at the $(h+1)^{\text {th }}$ step of iteration process, the updated parameter is obtained as 


$$
\hat{\lambda}^{(h+1)}=\hat{\lambda}^{(h)}-\frac{\left.\frac{\partial \log \pi(\lambda \mid \tilde{\boldsymbol{w}})}{\partial \lambda}\right|_{h}}{\left.\frac{\partial^{2} \log \pi(\lambda \mid \tilde{\boldsymbol{w}})}{\partial \lambda^{2}}\right|_{h}}
$$

where the notation $\left.A\right|_{h}$, for any partial derivative $A$, means the partial derivative evaluated at $\hat{\lambda}^{(h)}$. The second-order derivative of $\log \pi(\lambda \mid \tilde{\boldsymbol{w}})$ with respect to the parameter, required for proceeding with the Newton-Raphson method, is obtained as:

$$
\frac{\partial^{2} \log \pi(\lambda \mid \tilde{\boldsymbol{w}})}{\partial \lambda^{2}}=-\frac{m+a-1}{\lambda^{2}}+\sum_{i=1}^{n}\left\{\frac{\int x^{5} e^{-\lambda x^{2}} \mu_{\tilde{x}_{i}}(x) d x}{\int x e^{-\lambda x^{2}} \mu_{\tilde{x}_{i}}(x) d x}-\left[\frac{\int x^{3} e^{-\lambda x^{2}} \mu_{\tilde{x}_{i}}(x) d x}{\int x e^{-\lambda x^{2}} \mu_{\tilde{x}_{i}}(x) d x}\right]^{2}\right\}
$$

The iteration process then continues until convergence, i.e., until $\left|\hat{\lambda}^{(h+1)}-\hat{\lambda}^{(h)}\right|<\varepsilon$, for some pre-fixed $\varepsilon>0$.

\section{Method of moments}

Let $X$ be a random variable which has the Rayleigh distribution with pdf given by (1). It is known that the $k^{\text {th }}$ moment of the Rayleigh model with parameter $\lambda$ is

$$
E\left(X^{k}\right)=\Gamma\left(1+\frac{k}{2}\right) \lambda^{-\frac{k}{2}}
$$

Equating the first sample moment to the corresponding population moment, the following equation can be used to find the estimate of moment method:

$$
\lambda=\frac{\pi n^{2}}{4}\left\{\sum_{i=1}^{m} E_{\lambda}\left(X \mid \tilde{x}_{i}\right)+\sum_{i=1}^{m} \sum_{j-1}^{R_{i}} E_{\lambda}\left(Z \mid \tilde{z}_{i j}\right)\right\}^{-2} .
$$

Because the closed form of the solution to (20) could not be obtained, an iterative numerical process to obtain the parameter estimate is described as: 


\section{INFERENCE FOR THE RAYLEIGH DISTRIBUTION}

1. Let the initial estimate of $\lambda$ be $\lambda^{(0)}$, with $h=0$.

2. In the $(h+1)^{\text {th }}$ iteration, first compute

$$
E_{1 i}=E_{\lambda^{(h)}}\left(X \mid \tilde{x}_{i}\right)=\frac{\int x^{2} e^{-\lambda^{(h)} x^{2}} \mu_{\tilde{x}_{i}}(x) d x}{\int x e^{-\lambda^{(h)} x^{2}} \mu_{\tilde{x}_{i}}(x) d x}, \quad i=1, \ldots, m,
$$

and

$$
E_{2 i}=E_{\lambda^{(h)}}\left(Z \mid \tilde{z}_{i j}\right)=\frac{\int z^{2} e^{-\lambda^{(h)} z^{2}} \mu_{\tilde{z}_{i j}}(z) d z}{\int z e^{-\lambda^{(h)} z^{2}} \mu_{\tilde{z}_{i j}}(z) d z}, i=1, \ldots, m, j=1, \ldots, R_{i} .
$$

The new estimate of $\lambda$, for example $\lambda^{(h+1)}$, can be obtained from:

$$
\lambda^{(h+1)}=\frac{\pi n^{2}}{4}\left\{\sum_{i=1}^{m}\left(E_{1 i}+R_{i} E_{2 i}\right)\right\}^{-2} .
$$

3. Checking convergence, if the convergence occurs then the current $\lambda^{(h+1)}$ is the estimate of $\lambda$ by the method of moments; otherwise, set $h=h+1$ and go to Step 2 .

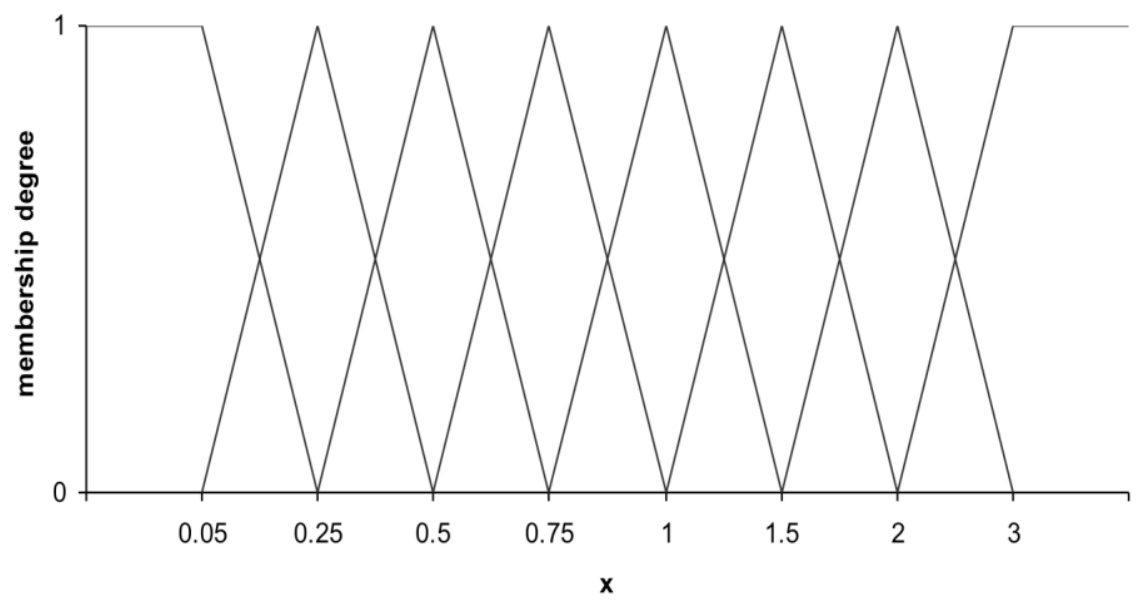

Figure 1. Fuzzy information system used to encode the simulated data 


\section{PAK ET AL}

\section{Numerical Study}

Experimental results illustrate how the different methods behave for varying sample sizes. First, for fixed $\lambda=1$ and different choices of $n, m$ and censoring scheme $\left(R_{1}, \ldots, R_{m}\right)$, progressively censored samples from the Rayleigh distribution were generated, using the method proposed by Balakrishnan and Sandhu (1995), as follows:

1. Generate $Z_{i}$ from $U(0,1)$ for $i=1, \ldots, m$.

2. For given values of the progressive censoring scheme $\left(R_{1}, \ldots, R_{m}\right)$, set $V_{i}=Z_{i}^{1 / a_{i}}, a_{i}=i+\sum_{j=m-i+1}^{m} R_{j}, i=1, \ldots, m$.

3. Set $U_{i}=1-V_{m-i+1} V_{m-i+2} \ldots V_{m}, i=1, \ldots, m$.

4. Thus, $X_{i}=F^{-1}\left(U_{i}\right), i=1, \ldots, m$, is the desired progressive type-II censored sample from the Rayleigh distribution.

Each realization of $\boldsymbol{x}$ was then fuzzified using the f.i.s. shown in Figure 1, and the ML, HPD and moment estimates (MME) of $\lambda$ for the fuzzy sample were computed using the methods provided in the preceding sections. For computing the HPD estimate of the unknown parameter, assume that $\lambda$ has $\operatorname{Gamma}(a, b)$ prior. To make the comparison meaningful, it is assumed that the priors are noninformative, and they are $a=b=0$. Note that in this case the priors are nonproper also. Press (2001) suggested using very small non-negative values of the hyperparameters in this case, and it will make the priors proper. This study uses $a=b=0.0001$. The results are not significantly different than the corresponding results obtained using non-proper priors, and are not reported due to space. The average values and mean squared errors of the estimates, computed over 1,000 replication, are presented in Tables 1-3. 


\section{INFERENCE FOR THE RAYLEIGH DISTRIBUTION}

Table 1. Average value (AV) and mean squared error (MSE) of the estimates of $\lambda$ for different censoring schemes. $(n=20)$

\begin{tabular}{cccccccc}
\hline \multirow{2}{*}{$\begin{array}{c}\text { Censoring } \\
\text { scheme }\end{array}$} & \multicolumn{2}{c}{ MLE } & \multicolumn{2}{c}{ HPD } & \multicolumn{2}{c}{ MME } \\
\hline \multirow{2}{*}{8} & $(0, \ldots, 0,12)$ & 1.181 & 0.219 & 1.163 & 0.185 & 1.184 & 0.221 \\
& $(12,0, \ldots 0)$ & 1.178 & 0.211 & 1.159 & 0.173 & 1.175 & 0.207 \\
& $(0,12,0, \ldots, 0)$ & 1.163 & 0.203 & 1.151 & 0.169 & 1.160 & 0.202 \\
& & & & & & & \\
10 & $(0, \ldots, 0,10)$ & 1.140 & 0.172 & 1.127 & 0.148 & 1.141 & 0.172 \\
& $(10,0, \ldots 0)$ & 1.153 & 0.182 & 1.136 & 0.166 & 1.155 & 0.184 \\
& $(0,10,0, \ldots, 0)$ & 1.145 & 0.177 & 1.130 & 0.154 & 1.148 & 0.179 \\
& & & & & & & \\
15 & $(0, \ldots, 0,5)$ & 1.127 & 0.155 & 1.114 & 0.132 & 1.122 & 0.151 \\
& $(5,0, \ldots 0)$ & 1.132 & 0.161 & 1.119 & 0.137 & 1.130 & 0.159 \\
& $(0,5,0, \ldots, 0)$ & 1.138 & 0.164 & 1.125 & 0.140 & 1.133 & 0.162 \\
\hline
\end{tabular}

Table 2. Average value (AV) and mean squared error (MSE) of the estimates of $\lambda$ for different censoring schemes. $(n=30)$

\begin{tabular}{cccccccc}
\hline \multirow{2}{*}{$m$} & $\begin{array}{c}\text { Censoring } \\
\text { scheme }\end{array}$ & AV & MSE & AV & MSE & AV & MSE \\
\hline \multirow{2}{*}{8} & $(0, \ldots, 0,12)$ & 1.163 & 0.185 & 1.149 & 0.162 & 1.166 & 0.187 \\
& $(12,0, \ldots 0)$ & 1.167 & 0.188 & 1.152 & 0.169 & 1.165 & 0.184 \\
& $(0,12,0, \ldots, 0)$ & 1.155 & 0.179 & 1.143 & 0.157 & 1.152 & 0.170 \\
& & & & & & & \\
10 & $(0, \ldots, 0,10)$ & 1.138 & 0.160 & 1.127 & 0.145 & 1.137 & 0.163 \\
& $(10,0, \ldots 0)$ & 1.125 & 0.154 & 1.119 & 0.130 & 1.123 & 0.147 \\
& $(0,10,0, \ldots, 0)$ & 1.132 & 0.159 & 1.122 & 0.138 & 1.130 & 0.156 \\
& & & & & & & \\
15 & $(0, \ldots, 0,5)$ & 1.112 & 0.136 & 1.103 & 0.117 & 1.114 & 0.139 \\
& $(5,0, \ldots 0)$ & 1.116 & 0.138 & 1.105 & 0.125 & 1.119 & 0.131 \\
& $(0,5,0, \ldots, 0)$ & 1.121 & 0.133 & 1.109 & 0.115 & 1.120 & 0.133 \\
\hline
\end{tabular}

Table 3. Average value (AV) and mean squared error (MSE) of the estimates of $\lambda$ for different censoring schemes. $(n=50)$

\begin{tabular}{cccccccc}
\hline \multirow{2}{*}{$m$} & $\begin{array}{c}\text { Censoring } \\
\text { scheme }\end{array}$ & \multicolumn{2}{c}{ MLE } & \multicolumn{2}{c}{ HPD } & \multicolumn{2}{c}{ MME } \\
& \multirow{2}{*}{8} & MSE & AV & MSE & AV & MSE \\
\hline & $(0, \ldots, 0,12)$ & 1.134 & 0.115 & 1.124 & 0.098 & 1.137 & 0.118 \\
& $(12,0, \ldots 0)$ & 1,141 & 0.122 & 1.130 & 0.113 & 1.145 & 0.125 \\
& $(0,12,0, \ldots, 0)$ & 1.139 & 0.119 & 1.127 & 0.105 & 1.136 & 0.114 \\
\multirow{2}{*}{10} & $(0, \ldots, 0,10)$ & 1.085 & 0.097 & 1.062 & 0.071 & 1.082 & 0.095 \\
& $(10,0, \ldots 0)$ & 1.079 & 0.093 & 1.056 & 0.065 & 1.077 & 0.090 \\
& $(0,10,0, \ldots, 0)$ & 1.073 & 0.088 & 1.051 & 0.059 & 1.070 & 0.084 \\
& & & & & & & \\
15 & $(0, \ldots, 0,5)$ & 1.038 & 0.069 & 1.021 & 0.037 & 1.042 & 0.072 \\
& $(5,0, \ldots 0)$ & 1.025 & 0.045 & 1.011 & 0.026 & 1.025 & 0.053 \\
& $(0,5,0, \ldots, 0)$ & 1.031 & 0.062 & 1.018 & 0.031 & 1.036 & 0.066 \\
\hline
\end{tabular}




\section{PAK ET AL}

Several points are clear from the experiment: Even for small sample sizes, the performances of the estimates are satisfactory in terms of AVs and MSEs. For all the methods, it is observed that for fixed $n$ as $m$ increases, the MSEs of the estimates decrease. Among the three estimation procedures developed in the paper, the HPD procedure gives the most precise parameter estimates as shown by MSEs in Tables 1-3.

\section{Application example}

To demonstrate the application of proposed methods to real data, consider the data collected during the experiment reported by Pak et al. (2013). In this experiment, a sample of 25 ball bearings is placed on a life test. A ball bearing may work perfectly over a certain period but be breaking for some time and finally be unusable at a certain time. So, the observed failure times of the ball bearings are reported by fuzzy numbers $\tilde{x}_{i}=\left(a_{i}, x_{i}, b_{i}\right)$, where $a_{i}=0.05 x_{i}$ and $b_{i}=0.03 x_{i}$ with the membership functions

$$
\mu_{\tilde{x}_{i}}(x)=\left\{\begin{array}{ll}
\frac{x-\left(x_{i}-a_{i}\right)}{a_{i}} & x_{i}-a_{i} \leq x \leq x_{i}, \\
\frac{x_{i}+b_{i}-x}{b_{i}} & x_{i} \leq x \leq x_{i}+b_{i},
\end{array} \quad i=1, \ldots, 25 .\right.
$$

Progressively censored samples of size $m=10$ were considered from these fuzzy data using three different sampling schemes, namely:

Scheme 1: $R_{1}=\ldots=R_{m-1}=0$ and $R_{m}=15$.

Scheme 2: $R_{1}=15$ and $R_{2}=\ldots=R_{m}=0$.

Scheme 3: $R_{1}=\ldots=R_{m-1}=1$ and $R_{m}=6$.

the estimate of the parameter $\lambda$ was then computed using the ML, HPD and moment methods. For computing the HPD estimates, it was assumed that $\lambda$ has $\operatorname{Gamma}(a, b)$ prior, including the non-informative gamma prior, i.e. $a=b=0$ and informative gamma prior, i.e. $a=b=2$. All the results are summarized in Table 4 . 


\section{INFERENCE FOR THE RAYLEIGH DISTRIBUTION}

Table 4. ML, HPD and moment estimates of the parameter for Example 2

\begin{tabular}{ccccc} 
Scheme & MLE & MME & HPD $(\boldsymbol{a}=\boldsymbol{b}=\mathbf{0})$ & HPD $(\boldsymbol{a}=\boldsymbol{b}=\mathbf{2})$ \\
\hline 1 & 0.00016 & 0.00015 & 0.00027 & 0.00033 \\
2 & 0.00043 & 0.00048 & 0.00058 & 0.00061 \\
3 & 0.00019 & 0.00021 & 0.00032 & 0.00039 \\
\hline
\end{tabular}

\section{Conclusions}

Some work has been done in the past on the estimation of the parameter of Rayleigh distribution based on complete and censored samples, but traditionally it is assumed that the data available are performed in exact numbers. In real world situations, however, some collected lifetime data might be imprecise and are represented in the form of fuzzy numbers. Therefore, suitable statistical methodology is needed to handle these data. This article proposed different procedures for estimating the parameter of Rayleigh distribution under progressive type-II censoring when the available observations are described by means of fuzzy information. They are maximum likelihood estimation (MLE), highest posterior density (HPD) estimation and method of moments (MME). A simulation study was conducted to assess the performance of these procedures. Based on the results of the simulation study, it may be observed that, the performance of the HPD estimates is generally best followed by the MLE and MME. Thus, it would seem reasonable to recommend the use of the HPD procedure for estimating the unknown parameter $\lambda$ from the Rayleigh distribution.

\section{References}

Azimi, R., Yaghmaei, F., \& Azimi, D. (2012). Comparison of Bayesian estimation methods for Rayleigh progressive censored data under the different asymmetric loss function. International Journal of Applied Mathematical Research, 1(4): 452-461.

Balakrishnan, N. (2007). Progressive censoring methodology: an appraisal. Test, 16(2): 211-296.

Balakrishnan, N. \& Aggarwala, R. (2000). Progressive censoring, theory, methods and applications. Boston, MA: Birkhauser. 


\section{PAK ET AL}

Balakrishnan, N. \& Sandhu, R. A. (1995). A simple algorithm for generating progressively Type-II censored samples. The American Statistician, 49(2): 229230.

Bhattacharya, S. K., \& Tyagi, R. K. (1990). Bayesian survival analysis based on the Rayleigh model. Trabajos de Estadistica, 5: 81-92.

Chung, Y. (1995). Estimation of scale parameter from Rayleigh distribution under entropy loss. Journal of Applied Mathematics and Computing, 2(1): 33-40.

Denoeux, T. (2011). Maximum likelihood estimation from fuzzy data using the EM algorithm. Fuzzy Sets and Systems, 183(1): 72-91.

Dey, S., \& Maiti, S. S. (2012). Bayesian estimation of the parameter of Rayleigh distribution under the extended Jeffrey's prior. Electronic Journal of Applied Statistical Analysis, 5(1), 44-59.

Dubois, D., \& Prade, H. (1980). Fuzzy Sets and Systems: Theory and Applications. Academic Press, New York.

Dyer, D. D. \& Whisenand, C. W. (1973). Best linear unbiased estimator of the parameter of the Rayleigh distribution. IEEE Transactions on Reliability $R$ 22: 27-34, 455-466.

Fernandez, A. J. (2010). Bayesian estimation and prediction based on Rayleigh sample quantiles. Quality \& Quantity, 44: 1239-1248.

Kim, C., and Han, K. (2009). Estimation of the scale parameter of the Rayleigh distribution under general progressive censoring. Journal of the Korean Statistical Society, 38: 239-246.

Lee, W., Wu, J., Hong, M., Lin, L., \& Chan, R. (2011). Assessing the lifetime performance index of Rayleigh products based on the Bayesian estimation under progressive type II right censored samples. Journal of Computational and Applied Mathematics, 235: 1676-1688.

Krishna, H. \& Kumar, K. (2011). Reliability estimation in Lindley distribution with progressively type-II right censored sample. Mathematics and Computers in Simulation, 82: 281-294.

Pak, A., Parham, G. A., \& Saraj, M. (2013). On estimation of Rayleigh scale parameter under doubly type-II censoring from imprecise data. Journal of Data Science, 11: 303-320.

Pradhan, B., \& Kundu, D. (2009). On progressively censored generalized exponential distribution. Test, 18: 497-515. 


\section{INFERENCE FOR THE RAYLEIGH DISTRIBUTION}

Raqab, M. Z., \& Madi, T. M. (2011). Inference for the generalized Rayleig distribution based on progressively censored data. Journal of Statistical Planning and Inference, 141: 3313-3322.

Rastogi, M. K., \& Tripathi, Y. M. (2012). Estimating the parameters of Burr distribution under progressive type II censoring. Statisticak Methodology, 9: 381391.

Rayleigh, J. W. S. (1880). On the resultant of a large number of vibration of the somepith and of arbitrary phase. Philosophical Magazine, 5th series, 10: 7378.

Press, S. J. (2001). The subjectivity of scientists and the Bayesian approach. New York: Wiley.

Tanaka, H. Okuda, T. \& Asai, K. (1979). Fuzzy information and decision in statistical model. In M. M. Gupta et al., Eds. Advances in Fuzzy Sets Theory and Applications, pp. 303-310. Amsterdam: North-Holland Publishing Co.

Zadeh, L. A. (1968). Probability measures of fuzzy events. Journal of Mathematical Analysis and Applications 10: 421-427. 